\title{
ICT Driven Smart Lighting Solution "iLIGHT" for Smart Cities: A Conceptual Framework
}

\author{
Dipak S. Gade \\ Post-Doctoral Research Scholar, Department of Computer Science and Engineering, \\ Srinivas University, Mangalore, India \\ ORCID ID: 0000-0002-0962-0375, Email: dipak.pdf@ srinivasuniversity.edu.in
}

Subject Area: Computer Science.

Type of the Paper: Conceptual Analysis.

Type of Review: Peer Reviewed as per $|\mathrm{C}| \mathrm{O}|\mathrm{P}| \mathrm{E} \mid$ guidance.

Indexed In: OpenAIRE.

DOI: https://doi.org/10.5281/zenodo.5506812

Google Scholar Citation: IJAEML

\section{How to Cite this Paper:}

Gade, Dipak S., (2021). ICT Driven Smart Lighting Solution "iLIGHT" for Smart Cities: A Conceptual Framework. International Journal of Applied Engineering and Management Letters (IJAEML), 5(2), 78-95. DOI: https://doi.org/10.5281/zenodo.5506812

International Journal of Applied Engineering and Management Letters (IJAEML) A Refereed International Journal of Srinivas University, India.

Crossref DOI : https://doi.org/10.47992/IJAEML.2581.7000.0105

(C) With Authors.

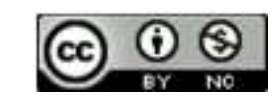

This work is licensed under a Creative Commons Attribution-Non-Commercial 4.0 International License subject to proper citation to the publication source of the work.

Disclaimer: The scholarly papers as reviewed and published by the Srinivas Publications (S.P.), India are the views and opinions of their respective authors and are not the views or opinions of the S.P. The S.P. disclaims of any harm or loss caused due to the published content to any party. 


\title{
ICT Driven Smart Lighting Solution "iLIGHT" for Smart Cities: A Conceptual Framework
}

\author{
Dipak S. Gade \\ Post-Doctoral Research Scholar, Department of Computer Science and Engineering, \\ Srinivas University, Mangalore, India \\ ORCID ID: 0000-0002-0962-0375, Email: dipak.pdf@ srinivasuniversity.edu.in
}

\begin{abstract}
Purpose: Smart Cities offers enhanced quality and performance of urban services using various ICT and Digital Technologies. It is a fact that more and more people are migrating to Smart Cities for better career opportunities and to have an enjoyable and comfortable life. With the ever-increasing population, Smart Cities always need to do better capacity management to best utilize and optimize the usage of available resources. One of such challenges faced by Smart Cities is the generation of electricity to meet the increasing demand for lighting. The objective is to have an efficient as well as an economical lighting system that can provide sufficient lighting to its infrastructure facilities, offices, Smart Buildings, Smart Parks, and Public properties. In this paper, I have proposed ICT based Smart Lighting Solution "iLIGHT", which is capable of meeting the lighting need of Smart Cities in a more efficient and economical manner. The advantages and limitations of "iLIGHT" are also discussed in detail. The analytics-based test results revealed that "iLIGHT" can reduce power consumption by almost 30\%. I have also provided the summary of reviewed existing literature on the lighting solutions used by Smart Cities and the advantages and limitations of these lighting solutions. I also analysed different usage scenarios for indoor and outdoor Smart Lighting Systems in Smart Cities and their typical power consumption. Finally, I have covered information with respect to my future plans on the "iLIGHT" Lighting Solution.
\end{abstract}

Design/Methodology/Approach: A systematic literature review is carried out for relevant white papers, data presented over relevant websites, available research papers and conference papers on Smart Lighting System for Smart Cities. I also interacted with SMEs and Industry professionals working on this research area. The collected research material is then properly studied and analysed to identify new facts and requirements related to Smart Lighting System. Based on the new findings and data analysis results, a new Smart Lighting Solution "iLIGHT" is proposed for Smart City Smart Lighting System.

Findings/Result: Smart Lighting System for Smart City is a complex system and used not only for providing efficient lighting across the Smart City but also used to interconnect various smart devices across Smart City and to provide seamless data interactions across the connected products. The Proposed "ILIGHT" Solution for Smart Lighting System in Smart City, is safe, reliable, and less expensive in design and deployment. It can offer good energy efficient Lighting System for Smart City and if used with proper configuration settings then it can be extremely fast and economical to operate and maintain.

Originality/Value: The information presented in this paper with respect to proposed "iLIGHT" Solution is original and genuine and is an outcome of interactions with SMEs, Smart City stakeholders and study and analysis of various research papers in order to understand the advantages and limitations of various Smart Lighting Systems used in Smart Cities across the globe. The paper has provided new findings with respect to Smart Lighting Systems for Smart Cities, and they are completely based on own data analysis and proposed new iLIGHT Solution.

Paper Type: Technology oriented Research

Keywords: Smart City, IoT, ICT, Data Analytics, SLS, IoL 


\section{INTRODUCTION :}

Smart Cities are continuously evolving and becoming smarter by adopting latest technology trends, ICT driven solutions and high-tech infrastructure [1]. To run a complex networked infrastructure, day to day operations and services, Smart Cities needs tremendous amount of electricity. Apart from infrastructure, the other area where power consumption is high is Lighting System. Meeting ever increasing power demand is one of the big challenges in front of Smart Cities. To make their residents life comfortable and more enjoyable many Smart Cities services run day and night, causing more demand of electricity. Thanks to the latest digital technologies and infrastructure that needs lesser power. In similar way, Smart City researchers are finding out many new solutions to make Smart City Lighting System more energy efficient and smarter. To deal with higher energy consumption related issue, many Smart Cities started using Light Emitting Diode (LED) Lights in place of traditional Tube Lights and normal bulbs. In Smart Cities, almost 50\% of city's entire energy cost is due to power consumed by Street Lights. So just imagine how much savings one can have after using LED Lights. Just by using LED Lights in place of traditional lights, it is estimated that New York City will save \$14 Million per year whereas Chicago City is estimated to save 10 million US Dollars per year [2]. There are various types of LED Lights which can be used for different purposes across the Smart City. Different types of LED lights are stated in Table 1 along with their purpose and description. Also, refer figure 1 which has shown the different types of LED Lights. Note that the advantage of using LED Lights is they can be powered from electricity source which has been obtained using Solar, Wind, or other renewable energy sources.

Table 1: Typical LED Light Types

\begin{tabular}{|c|c|c|}
\hline $\begin{array}{l}\text { Sr. } \\
\text { No. }\end{array}$ & Type of LED Light & Purpose \\
\hline 1. & LED Street Light & $\begin{array}{l}\text { Used for lighting City streets and roads. Typically installed on a } \\
\text { heighted pole which are mounted across the streets and roads. } \\
\text { Covers wide area for lighting. }\end{array}$ \\
\hline 2. & $\begin{array}{l}\text { LED High Bay } \\
\text { Lights }\end{array}$ & $\begin{array}{l}\text { These LED Lights are indoor LED Lights and use for lighting } \\
\text { industrial complex, warehouses where ceiling height is around } 20 \\
\text { to } 40 \text { feet. }\end{array}$ \\
\hline 3. & LED Garden Lights & $\begin{array}{l}\text { As name suggests, these lights are used to light Garden Area. The } \\
\text { LED Lights are installed on a medium and short heighted pole } \\
\text { which are mounted in distributed areas. These Lights covers short } \\
\text { and medium area of Garde }\end{array}$ \\
\hline 4. & LED Flood Lights & $\begin{array}{l}\text { These are wide angle LED Lights used to cover a large area or } \\
\text { "flood" the area with light. Mostly use in outdoor lighting e.g., to } \\
\text { light playground. It produces a very high intensity of light }\end{array}$ \\
\hline 5. & LED Lawn Lights & $\begin{array}{l}\text { These are outdoor LED Lamps typically used to light smaller areas } \\
\text { like lawns, porch etc. These LED lamps are having low power } \\
\text { capacity }\end{array}$ \\
\hline 6. & LED Ceiling Lights & $\begin{array}{l}\text { These are indoor LED Lights, typically used for house, offices etc. } \\
\text { As name suggests they are mounted in ceilings and come in variety } \\
\text { of shapes and sizes. }\end{array}$ \\
\hline 7. & LED Panel Lights & $\begin{array}{l}\text { These are indoor LED Lights, typically used for Industrial shades, } \\
\text { Offices, ATMs etc. They mostly come in circular or rectangular } \\
\text { size and have flat panel kind of look. }\end{array}$ \\
\hline 8. & LED Linear Lights & $\begin{array}{l}\text { These Lights are formed by many Light Emitting Diodes packaged } \\
\text { together in a narrow long housing to have a strip of light. These } \\
\text { Lights are installed as either surface mounted to a wall or } \\
\text { suspended from a ceiling, or recessed into a wall or ceiling }\end{array}$ \\
\hline 9. & LED Strip Lights & $\begin{array}{l}\text { LED Strip Light is prepared by populating strip of LEDs on a } \\
\text { flexible circuit board that can stick anywhere and bend easily and } \\
\text { can add powerful lighting in a bright variety of colours }\end{array}$ \\
\hline 10. & LED Spot Lights & $\begin{array}{l}\text { As the name suggests these LED Lights are used for lighting a } \\
\text { narrow beam or for spotlight, typically used in LED Torch and for } \\
\text { surveillance purpose }\end{array}$ \\
\hline
\end{tabular}


Using LED lights in place of conventional lights can reduce the power consumption, however if LED lights can be turn on and off in a controlled manner then it can further optimize the power usage. There are three different types of controlling LED Lights, each can offer significant advantage and benefits and have certain limitations as well. Refer table 2, which has stated comparison among three types of LED Lights controls with respected to its operating features, benefits, and limitations of each lighting control scheme. As clear from Table 2, LED Lights can reduce power consumption but just using LED Lights is not a smart and or intelligent solution, it cannot provide any other useful information such as power consumption statistics. To get the best out of LED Lights it is also necessary to have the functionalities like intelligently on/off control, brightness control, etc.

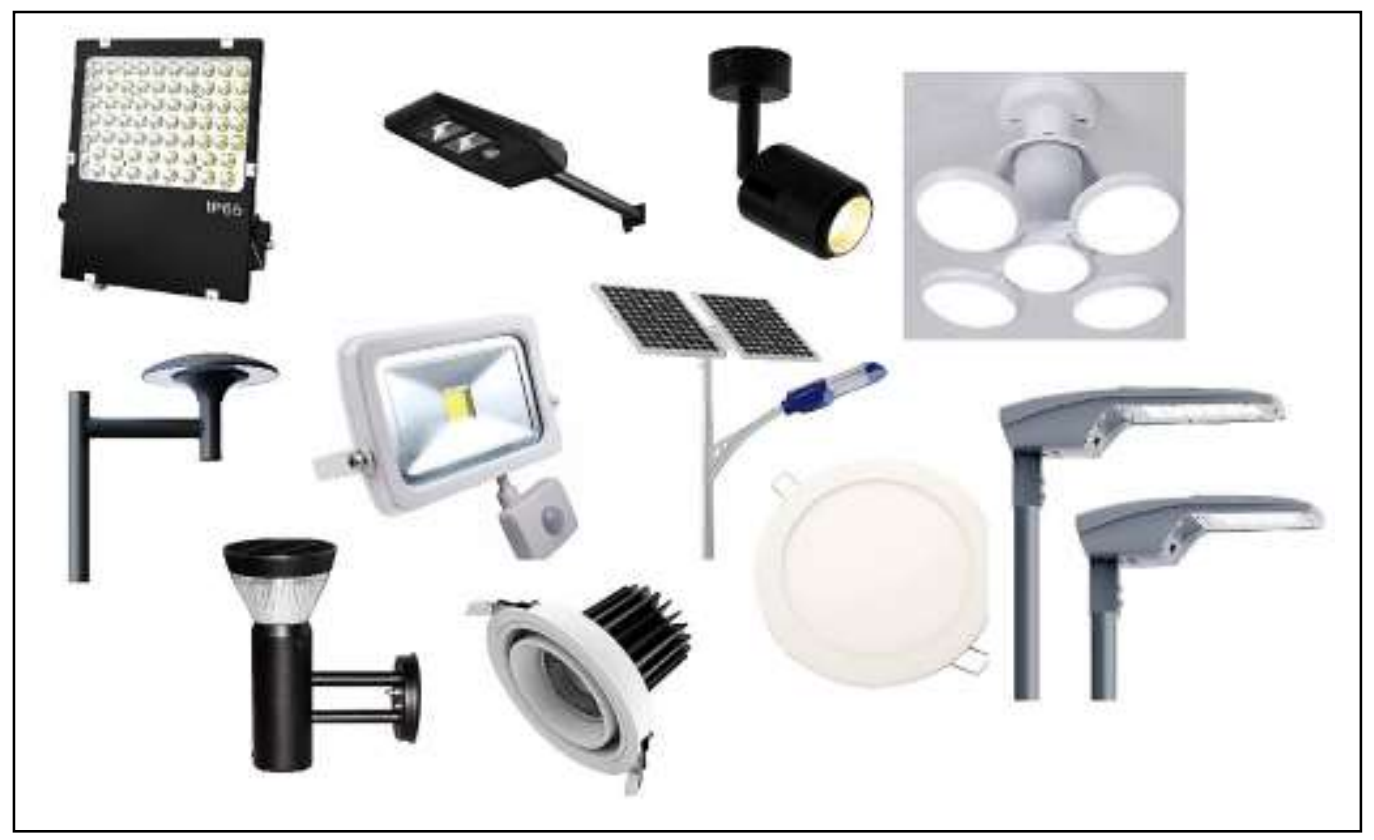

Fig. 1: Different LED Light Products used across Smart City (Source: nctled.com)

From the last few years, due to technology advances, all the conventional lighting systems are getting replaced by Smart Lighting System (SLS) that not only consumes less power but also provides an intelligent control and more lights with a small footprint. Smart Lighting System is a lighting technology for Smart Cities and Smart Homes that are connected to the Internet Network and can make intelligent decisions such as following lighting on/off/dim schedule based on predefined time or environmental conditions or traffic conditions. These lights are capable of remote controlled and or voice controlled as per need, the system also facilitates smart data analytics to provide various useful informatic such as power consumption, cost of running, fault predictions, maintenance schedule, and alerts.

Table 2: LED Light Control and management

\begin{tabular}{|c|c|c|c|}
\hline Feature/ Function & Manual Switching & $\begin{array}{c}\text { Connected LED } \\
\text { Lighting }\end{array}$ & $\begin{array}{c}\text { Self-Managed LED } \\
\text { Lighting }\end{array}$ \\
\hline Control & Manual & Automatic & Automatic and Intelligent \\
\hline Design & Simple & Complex & Complicated \\
\hline Operation & Simple & Easy & Easy \\
\hline Maintenance & Economical & Less Economical & Costly \\
\hline Cost & Low & High & Costly \\
\hline Power Consumption & Comparatively Higher & Low & Lowest \\
\hline Energy Savings & High & Higher & Highest \\
\hline Installation & Simple & Complex & Highest \\
\hline Return on & High & Higher & Safest \\
\hline Investment & Safe & Safe & \\
\hline Safety & & &
\end{tabular}


The Smart Lighting System allows gathering data from connected infrastructure using appropriate sensors throughout the city or geographies or across properties. For example, streetlights equipped with proximity sensors, cameras, or environment sensors, air quality sensors could assist in the management of smart cities. Sensors embedded in domestic light fixtures could enhance hazard detection or emergency calling. In summary Smart Lighting System can facilitate making Smart City safer place to live, visit and work and in turn gives better sense of security to its citizens.

The typical Smart Lighting System has following features.

- Lighting control based on need in different areas as per lighting needs

- Lighting control taking into consideration environmental conditions such as fog, rain, humid etc.

- Lighting control based on the time of day

- Real time data statistics based on past on/off control data, weather conditions, environmental conditions and predictions related to energy requirements

- Predict, faster detect, and address lighting outages related issues

- Brighter lights and control in remote areas or traffic dense areas

- Instant brightness control on highways and to assist emergency situations

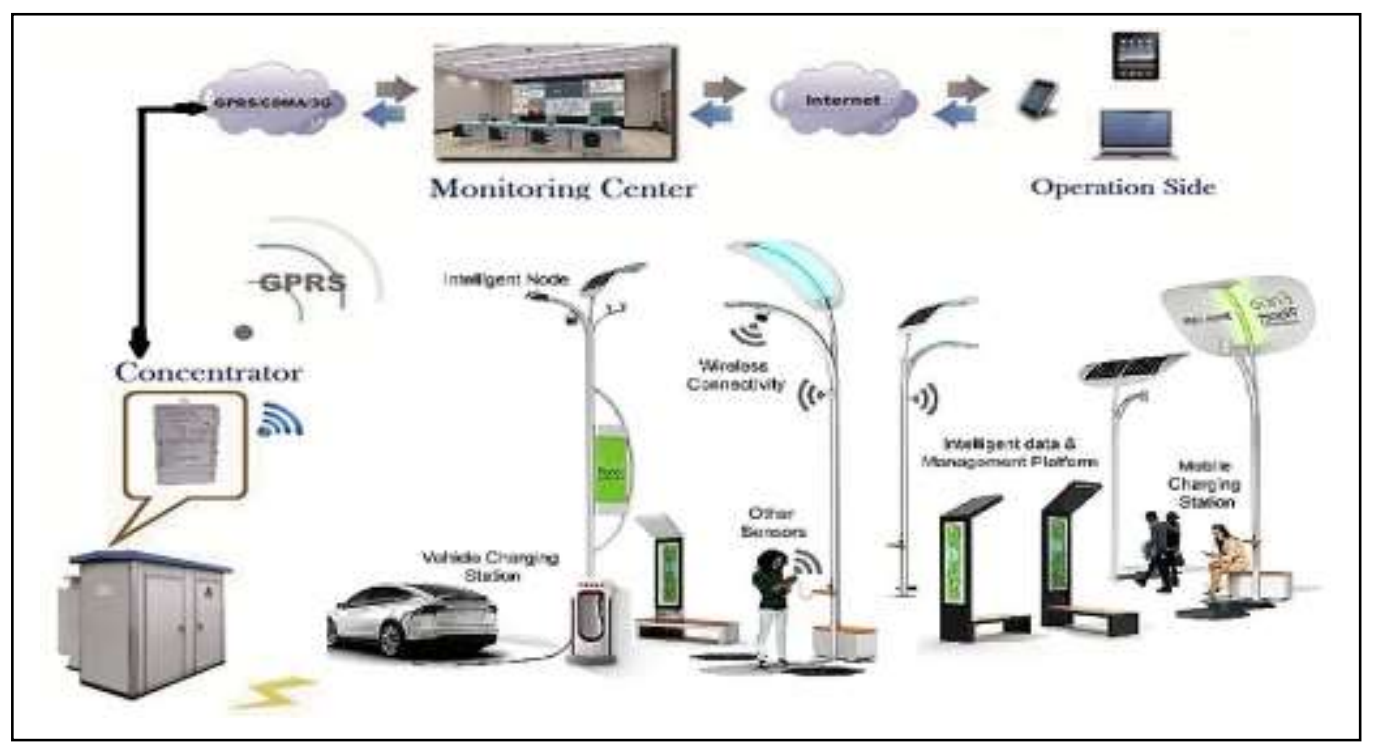

Fig. 2: Typical Smart Street Lighting for Smart City (Source: lightingindia.in, gaoterled.com)

Refer figure 2, which is showing a typical Smart Street Lighting System for Smart Cities. As shown in the figure, the smart street lighting system contains communication modules, lighting poles, light sensors, network modules, monitoring center, user interface apps etc. The conventional street lighting system keeps its operation limited to manually turn on and off lights. But the Smart Street Lighting System can do much more, for example dynamic turn on and turn off streetlights based on predefined conditions and or schedule, automatic light intensity controls, dimming of lights from remote, data analysis of power consumption, energy saved, operating cost etc. Smart Street Lighting System is part of Smart Lighting System and contains similar components and features which can be applicable for a Smart Lighting System.

\section{PROBLEM STATEMENT :}

Smart Cities are evolving day by day and their service portfolio is also expanding in order to offer better facilities to their residents. Smart Cities facilitate their residents with various services which can operate day and night. In order to have operations during night-time, Smart Cities need an effective and efficient lighting system. Based on operating areas, geographies, operating conditions, environmental conditions, seasonal variations, movements of City residents, traffic conditions, and types of coverage, Smart Cities need various kinds of lights and lighting arrangements. And, to power these lights, Smart Cities often need a tremendous amount of power and operating expenses. To deal with such challenges, Smart Cities use Smart Lighting systems. However, there are many challenges to design, operate and maintain the 
complex Smart Lighting System for Smart Cities. Unfortunately, the availability of ready reference design data for selecting or designing of such a Smart Lighting System is challenging. There are varieties of Smart Lighting Systems, and it is also difficult to understand the critical components like sensors, servers, application software, etc. to choose from while making a new Smart Lighting System. To deal with this problem, this Research paper is written with an intention to provide detailed reference information on Smart Lighting System and its key functionalities, benefits, and advantages. The reference framework for choosing the right Smart Lighting Solution is also provided to assist the stakeholders while designing/choosing the right Smart Lighting System.

\section{RESEARCH OBJECTIVES :}

This research paper has covered information related to various existing Smart Lighting systems for Smart Cities. With the available time constraints, limited infrastructure, and considering research scope, availability of literature, this research is carried out keeping in mind following broad objectives

- Study the existing Smart Lighting Systems of Smart Cities

- Analyse the data related to power consumption of the existing Smart Lighting Systems and related cost savings

- Identify advantages, limitations, and issues if any with the existing Smart Lighting Systems deployed in various Smart Cities.

- Propose the IoT and ICT based Smart Lighting Solution for Smart Cities

- Identify the improvement opportunities which can be carried out in the future for the proposed Smart Lighting Solution

- To provide a context and foundation for promoting new research activities on the Smart Lighting Solutions for Smart Cities

\section{METHODOLOGY :}

Exploring existing Smart Lighting Systems for Smart Cities is a bit complex and time-consuming task. A lot of secondary research data in the form of Journal research papers, conference papers, books, online research blogs, etc. is available on Smart Cities Lighting Systems covering architecture, operation, and maintenance related information. While preparing the existing research paper, the focus was on reviewing selected research papers from high-impact factor international and national research journals and research data available on some selected websites. While exploring the research data on Smart Cities Smart Lighting Systems Specific keywords such as "Smart City", "Smart Lighting System" "Smart City SLS", "Smart City Power Consumption", "Smart City Lights" were used. The collected research data was then further analysed considering the following questions and requirements

- How much manpower is required to maintain the Smart Lighting Systems?

- What is the operating cost to maintain the Smart Lighting Systems?

- How much power is consumed on a daily/monthly/yearly basis the Smart Lighting Systems?

- How much power is saved by the Smart Lighting system in comparison to using traditional lighting system?

- The complexity involved while designing and deploying the Smart Lighting Systems across Smart City

- The operational safety and reliability of the Smart Lighting Systems

Data are collected by interacting with various researchers, subject matter experts, and industry professionals working on Smart Lighting Systems and tried to understand their views specifically with respect to following

- Essential requirements which Smart Lighting Systems needs to fulfil

- Typical Technologies used while developing the Smart Lighting Systems

- Various Sensors were used to collect the relevant data

- Overall complexity involved in the development of Smart Lighting Systems

- What are the areas of improvement with existing Smart Lighting Systems?

- Which additional functionality they would like to have in the future Smart Lighting Systems

Based on the collected research data and analysis, finally, a proposed Smart Lighting Solution conceptual framework is developed to undertake improvements in Smart Lighting Systems that can 
facilitate easy deployment, operation, and maintenance of advanced Smart Lighting Solution. A future plan with respect to proposed "iLIGHT" Smart Lighting Solution is also stated.

\section{RELATED WORK :}

In Industry 4.0 era, with IoT, Cyber security, and various connected technologies, Smart City Lighting Systems are also evolving, and significant advancements have been seen from using conventional lighting systems containing Bulbs and Tube Lights to the present Smart Lighting System containing connected and or self-managing LED lighting system [3]. Thanks to the worldwide researchers and industry professionals who actively contributed to such advancements and for providing enough research material on this subject matter.

In a Smart Lighting System, LED lights are predominantly used because of their quick response, higher light intensity with less power consumption, and very small footprint. Besides, LED Lights can easily operate with DC power derived from renewable energy sources including Solar, Water, or Wind power converters. M. H. Toufiq Imam, Sina Afshari and Sandipan Mishra in their book: Smart Lighting control system [4] have clarified that in the last few years with technology advancements specifically to achieve energy efficiency and using renewable energy sources with the help of innovative solutions that Smart Lighting Systems have found applications from individual houses to Smart Buildings and Smart Grids to Smart Factories as well as Smart Cities. Thus, Smart Lighting System covers street lightings, interior lighting in office buildings, and lighting in Smart Homes.

Amit Kumar, Abbas Acar, et al., in their paper "IoT enabled Smart Lighting Systems for Smart Cities" have described in detail IoT-based Smart Lighting System [5]. Authors have defined Smart Lighting System as an intelligent and automatic lighting control system that can be managed in a distributed or centralized way by different IoT-based sensors and various IoT Communication protocols. Authors have also clarified that energy consumption control and efficient illumination system are the key concepts in Smart Lighting System and how IoT-enabled Smart Lighting Communication Protocols can be used for efficient power management. The authors proposed a generic communication model for SLS. Authors have also successfully demonstrated the evaluation of various SLSs in different indoor and outdoor lighting settings.

Giuseppe Cacciatore, Claudio Fiandrino, et al., in their paper "Cost Analysis of Smart Lighting Solutions for Smart Cities" have proposed an intelligent Smart Lighting System based on IoT and augmented lampposts capable of turning on, off, delayed turn on, and dimming light based on absence or presence and a number of people around [6]. Authors have claimed that in absence of a Smart Lighting System, municipalities spend almost $40 \%$ of their allocated budget on street lighting energy expenses. The authors evaluated the concept based on the custom simulator which facilitates deployment of lampposts in the real urban environment and such lampposts get activated by the proposed heuristics of the nearby citizens who walk or travel nearby the lampposts. Authors have also clarified that replacing traditional lights with LED Lights and with the dimming of light intensity using their lighting system deployed in the city of Luxembourg, realized the huge savings in energy cost in the first year of deployment itself.

Marc Füchtenhans, Eric Grosse \& Christoph Glock in their paper "Smart lighting systems: state-of-theart and potential applications in warehouse order picking" carried out a detailed literature review to analyse various technologies used and applications of Smart Lighting Systems [7]. The authors specifically stressed the applications of SLS in industrial environments including production and logistics and clarified that SLS in industrial environments has to fulfil some specific requirements based on working environments and operating conditions. The authors discussed in detail the example and application of SLS to improve the efficiency of warehouse order picking. Authors have also confirmed that SLS objective is not limited to energy savings and cost reduction, but SLS Applications can also serve the purpose of data transmitters, provide safety and security to City residents, can provide indoor positioning functionalities, and so on.

Thomas Werff, Harm Essen, and Berry Eggen in their paper "The impact of the internet of lighting on the office lighting value network" have discussed the concept of the Internet of Lighting (IoL) as a part of Smart Lighting System [8]. Authors have claimed that Lighting in offices cannot only provide illumination to office infrastructure, but it can also facilitate connecting people, devices, and office systems with each other and to the Internet and thereby creates the Internet of Light. Authors have clarified that IoL can facilitate sharing of data, standardization, IP to the end node, and Light as a Service. The authors proposed a layered value network model to demonstrate the IoL concept. 
Baoshi Sun, Qiaoli Zhang and Shi Cao in their research paper "Development and Implementation of a Self-Optimizable Smart Lighting System Based on Learning Context in Classroom" have proposed a Smart Lighting System for Classrooms [9]. It can control Correlated Colour Temperature (CCT), adjust brightness and illumination spread as per classroom needs. Authors have confirmed that illumination is an important environmental factor in Classrooms and adjusting the light settings as per students need can improve student's performance. The authors claimed that the proposed Smart Lighting System can configure 10 lighting modes and automatic switching as per school schedule. The authors also claimed that their proposed SLS framework can help in exploring the correlation between environmental parameters and student's performance.

Yusi Cheng, Chen Fang, et al., in their paper "Application of a Smart Lighting System Based on Distributed Wireless Sensor Networks" have highlighted that reducing energy consumption is one of the major objectives behind using Smart Lighting System [10]. By using proper sensors such as ambient light sensor, PIR Passive Infrared Sensor, Occupancy Sensors, Microwave Doppler Sensors, etc., and distributed wireless network using Zigbee Communication Protocols along with illumination light controls in Smart Lighting System, it is possible to avoid inefficient controlling of lights. Authors have claimed that they evaluated the proposed SLS Model based on various scenarios and parameters such as illuminance control, people floe density, room occupancy, number of people present in the room, and so on. They found that their Smart Lighting System when evaluated at Metro Station and office room has resulted in energy savings of $45 \%$ and $36 \%$ respectively. The authors feel that their test results are encouraging and can be beneficial for the development of Smart buildings.

Tran Anh Khoa, Le Mai Bao Nhu, et al., "Designing Efficient Smart Home Management with IoT Smart Lighting: A Case Study" in their research paper proposed IoT based Smart Lighting system for Smart Home Management [11]. Authors argued that Smart Home management functionalities such as real time monitoring, automated burglar alarms, guest attendance, light switches control, etc can be fulfilled via IoT based Smart Lighting System where data communication can be encrypted through SHA-256 algorithms to ensure information security. Authors claimed that the Smart Home Management prototype with an interface with Mobile App was installed and evaluated at Ton Duc Thang University, Vietnam and the evaluation results confirmed the clear benefits including, low cost and robust security for a smart home.

In summary, the Smart Lighting System is used to save energy without doing any compromise on lighting quality and illumination, more over it also facilitate switching of lights automatically, intelligently without any manual intervention and also manually as per users' requirements. From the literature review, it is also confirmed that Smart Lighting System also facilitates data transmission, data analytics, user interface over mobile app, predictive diagnostics, remote monitoring, and management of lighting controls.

\section{SMART LIGHTING SYSTEM: A DEEP DIVE :}

Let us further explore the Smart Lighting System for Smart Cities [12-24]. Refer figure 3 which has shown the typical architecture of Smart Lighting System. As shown in the figure, the system components are connected together through a wired or wireless communication which is secured through data encryption. The system mostly covers the entire city area.

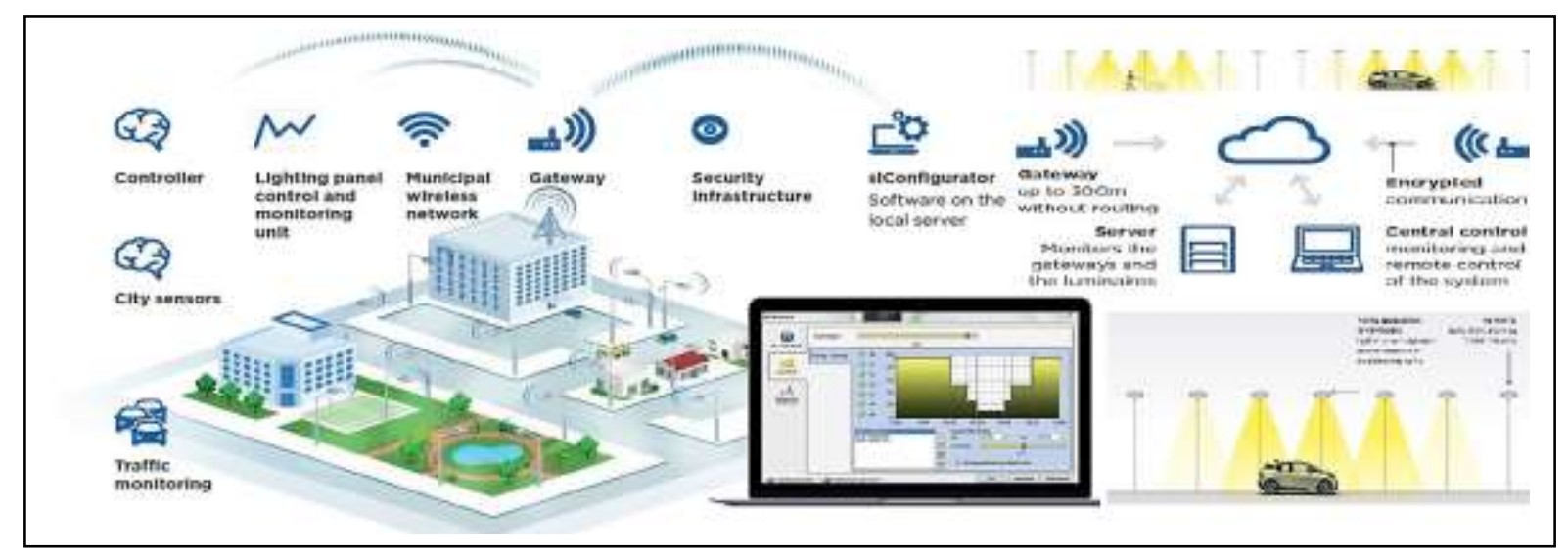

Fig. 3: Smart Lighting System for Smart City (Source: Lumenova.net) 
and or specific geographies. Existing IT infrastructure such as Servers, Display Monitors, Internet Gateway, Internet etc. of Smart City is normally used to host SLS Service. The components used in outdoor application are mostly at least IP58 complied and are of industrial grade quality to withstand harsh environment conditions. As shown in figure 3, the typical Smart Lighting System contains following major components.

\section{A. LED Lights:}

Smart Lighting System without LED Lights cannot be completed. We have already discussed various types of LED Lights which can be used across the Smart City. Based on the need and purpose, various LED Lights such as Street Lights, Spotlights, Flood Lights, Panel Lights etc. are used either indoor or outdoor areas for lighting purpose. LED Lights are advantageous due to their fast response, less power consumption and smaller footprint.

\section{B. Smart Sensors:}

Smart Sensors are IoT sensors capable of connecting over Internet. These Sensors are used to detect real time changes in information such as availability of natural light, weather conditions, environmental conditions, lamp inclination, air pollution, traffic conditions etc. The Sensors provides sensed data to the Smart Lighting System Server which runs with SLS Application Service and provides collected data analysis and visualization. These sensors can be of various types. Some selected types of IoT Sensors are as follows

- Temperature Sensors

- Humidity/Moisture Sensors

- Ambient Light Sensors

- Acoustic/Sound/Vibration Sensors

- Motion/Velocity/displacement Detector

- Thermal Camera

- CCTV Camera

\section{Communication Modules:}

There are mainly three types of communication systems used for implementing network connectivity within Smart Lighting System.

- Power Line Communications (PLC) : Here communication is realized using modulated communication signals over existing power distribution networks

- Broadband Power Line (BPL): BPL Network realizes the communication using the modulated data signals onto carriers in the megahertz range and higher

- Wireless Communication: Wireless communication modules are mostly installed on the Light Poles separately and or nearby LED Lights enclosure. As the name suggests, these modules provide capability to provide wireless communication with the Smart Lighting System Server and network devices. It may have RF communication or Bluetooth enabled low power wireless communication. The wireless communication is mostly encrypted using appropriate AES encryption algorithm.

The Communication Module provide bi-direction communication between SLS Network devices and LED Light Module and communicates the Sensor data and LED Lights ON/OFF and Light intensity related information. The wireless communication modules sometimes have an external antenna as well for better transmission and receiving of data and may also house GPS device to provide location coordinates.

\section{Gateway:}

Gateway is one of the important components in Smart Lighting System which primarily serves the purpose of connecting various end points of various SLS networks with each other and facilitates seamless communication across the Smart Lighting System network nodes. Typically, Gateway is capable of handling Gigabit network communication speed and is of industrial grade. Sometimes it is also called as IoT Gateway since it communicates data among various IoT enabled sensors of Smart Lighting System. It offers following key features

- It provides additional security.

- It facilitates communication bridging and M2M communication. 
- It offers device configuration and change management.

- Serves as a data cache, buffer, and streaming device.

- It facilitates offline services and real-time control of devices.

- It facilitates aggregates data, pre-processes, cleans, and filters data before sending it.

\section{E. SLS Server:}

SLS Server is one which hosts Smart Lighting System Service. It runs with SLS Application Software which contains communication module, Graphical User Interface, Cybersecurity Module, Data Analytics Module, Database Module, Authentication Module and Diagnostics Module. The SLS Server can be hosted on Cloud or it can be based on premises as per the need. It carries following major functionalities.

- Communication with various end points of SLS across Smart City including Streetlights, Indoor Lights, Traffic Lights, Parking Lights, Garden Lights, Ground Lights, etc.

- Storing of various end points data in secure encrypted fashion

- Carrying out various data analytics on the stored SLS end points data

- Authenticating Users as per SLS Policies

- Providing Web User Interface and Mobile App User Interface containing user dashboards and various data trends and important information related to power consumption, energy saved, diagnostics and fault related data, monitoring data, environmental data, etc.

- Provide manual ON/OFF control of LED Lights from various end points of SLS

- Provide relevant data information to other Servers of other Services of Smart Cities for data correlation and record

By using latest IoT Sensors, high tech IT infrastructure and powerful application software, it is possible for Smart Lighting Systems of Smart Cities to facilitate following features including but not limited to

- Full Lighting Control with Lights On/Off Scheduling, Dimming, Outage Notifications

- Data analytics and usage reports such as Power Consumption, Peak Power, Energy Saved, Energy required

- Remote Monitoring, Remote Switching of LED Lights, Manual Control of LED Lights

- Promotes use of renewable energy sources, Optimized Cost, Optimized power usage and thus overall cost savings

- With the help of various IoT Sensors, additional data information with respect to Air Quality, Wind Speed, Temperature Readings, Traffic Conditions, Location based Services

- Helps Smart City residents and visitors feel secure in their surroundings

- Predictive diagnostics by analysing abnormal conditions, functional defects, locating the malfunctioned lamps and scheduling preventive maintenance

\section{ILIGHT - A SMART LIGHTING SOLUTION :}

Smart Lighting System is a complex system and may be difficult to implement considering its wide scale and cross functional interface. After analysing the various existing Smart Lighting Systems and based on discussion with Industry SMEs and researchers, it was confirmed that besides energy savings, it is equally important to have Smart Lighting Systems easy to configure, install, deploy, operate, and maintain. Considering these key criteria, a conceptual framework called "iLIGHT" is proposed. Let us try to understand more about iLIGHT and how it works and can meet the end requirements.

iLIGHT is a conceptual framework of Smart Lighting Solution. Please refer to figure 4 which has presented high level block diagram of iLIGHT. It is a modular system which contains various blocks. The main building blocks of iLIGHT are as follows. 


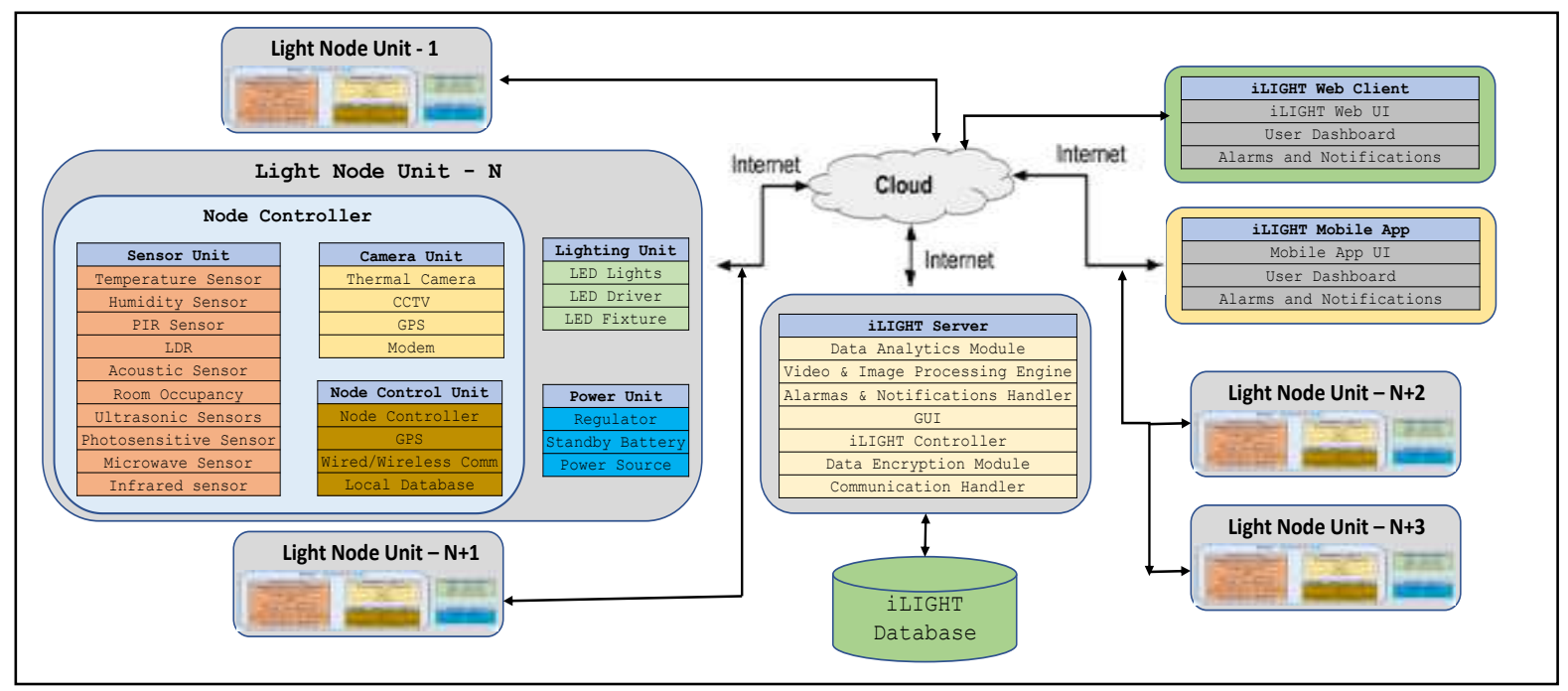

Fig. 4: Block Diagram of iLIGHT Smart Lighting Solution

\subsection{Light Node Unit (LNU)}

Light Node Unit is an essential element and one of the most important modules of iLIGHT Solution. LNU is installed at the required nodes such as street, office, building, home, ground, etc. and in some open places where the monitoring and or lighting area is wide. It acts as a monitoring and control unit and controls the switching operation of connected lights. It is also known as LNU. It contains the following main components

$>$ Sensor Unit : Sensor unit basically contains various sensors for sensing environmental conditions including Temperature, Humidity, Dust, Rain, Wind and also Ambient Light, People Movement, occupancy condition of the room, light switching, etc. The choice and selection of Sensors will be based on the area where these sensors need to be installed i.e., Street Light, Building, Lawn, Open Ground, Office, and or Home. Note that only selected sensors are listed down in the Block Diagram in figure 4, however, based on need the number and types of sensors can be easily increased or decreased. Note that these Sensors sense the relevant conditions and provide outcomes to the Node Control Unit for further processing. The Sensors may be housed in the enclosure of LNU or even can be installed outside the LNU enclosure suitably.

$>$ Node Control Unit (NCU) : This is an intelligent Unit and acts as the brain of LNU. It is also commonly referred to as NCU. NCU contains 32 bit Controller with suitable processing firmware. The job of NCU is to interact will all the local modules such as Sensor Unit, Camera Unit, Lighting Unit, and Power Unit and keeps health status information of each module. Process the relevant data from all these modules and communicate the processed information to the iLIGHT Server periodically. It also needs to share its own heart-bit information with the iLIGHT Server. Apart from this NCU is also proposed to equip with a sufficient local database of size around 32GB to last at least 1-week compact data captured by various sensors. The Local Database also stores the selected frame captured by CCTV and Thermal cameras after suitably zipping them. Note that the local database stored information is periodically sent to iLIGHT Server and once it is transferred then the Local Database trashes the stored information and keeps the storage available for freshly captured information from Sensors and Cameras. Each NCU has unique identification ID and mounted with GPS for informing its location coordinates to iLIGHT Server. The other important job NCU carries out is to securely communicate with iLIGHT Server with the available communication infrastructure i.e., over wired Ethernet or with wireless communication as the case may be. If the geography is already having a wired ethernet communication facility, then Modem or wireless communication module is not installed with such NCU, and NCU is directly wired with wired ethernet infrastructure. In case a Wired Internet connection is not available then NCU is equipped with a suitable Modem or Wireless Communication Module. Note that NCU is configured to send data periodically to iLIGHT Server and these communication packets can be sent by NCU as per configuration settings i.e., every 1 Minute and or in steps of 5 Minutes or as 
per commands from iLIGHT Server. NCU unit Firmware runs with a suitable AI algorithm to select or discard relevant Images and or Video footages and Sensor's data while sending it to iLIGHT. Note that NCU firmware is made capable enough to operate, manage and control its LNU even if NCU loses communication with iLIGHT Server. NCU also has Manual Override Settings and if it is active then the LNU can be completely operated with manual control bypassing NCU.

$>$ Camera Unit: In order to monitor the real-time conditions of specified geography/Building/Home and surrounding area, Thermal Cameras and CCTV Cameras are installed with the right mounting arrangement. These are mostly IP-based Cameras and are capable of communicating directly over the internet. The Camera Unit is also mounted with GPS and Modem to make it capable of directly communicating with the redundant and or alternate Node Controller or directly with iLIGHT Server in case the local Node Controller fails. In normal circumstances, the CCTV and Thermal Cameras output are fed to the local Node Controller. The local Node Controller can also adjust the Pan, Tilt, and Zoom settings of these cameras to ensure viewing the area as per the user's choice. Note that Thermal Cameras are recommended to install instead of normal cameras reason being thermal cameras have the capability to operate under sever environment conditions as well and can provide accurate video and images for processing purpose.

$>\quad$ Lighting Unit: As the name suggests Lighting Unit contains the LED Lights, their supporting mounting arrangements and fixtures, and LED Drivers. Note that the Lighting Unit is not an enclosed Unit as such. One Lighting Unit can contain various LED Lights as per the LNU geography need. It can contain multiple streetlights when LNU is installed at Street, or multiple Flood Lights, Spotlights, or Lawn Lights depending on if they need to be installed in Open Grounds, Lawns and Garden and or Playground. The lighting Unit is the most critical module of LNU and is responsible for providing the main functionality LNU. i.e. provides illumination as per the need of the stakeholders at the intended geography. The information related to the operation and maintenance status of the Lighting Unit is available with NCU and NCU is basically responsible for switching of LED Lights and controlling of illumination of the Lighting Unit as per the instructions from iLIGHT Server and or based on its own decision as the case may be. As stated earlier, it is possible to control manual switching of the Lighting Unit as per the configuration settings facilitated by NCU.

$>$ Power Unit: Power Unit serves the purpose of powering Electronic Control Circuit and LED Lights of LNU. Power Unit constitutes power converter which converts the power received from the renewable energy source such as Wind, Solar, Water, or mains source to DC Power. The derived DC Power is then regulated and stabilised in order to drive all the electronics components of LNU and LED Lights. Note that Power Unit typically also consists of rechargeable DC Battery which under normal scenario keeps charging and provides sufficient backup in case of power failure.

\section{2 iLIGHT Server}

This is the main important module of iLIGHT Smart Light Solution. It is the brain of iLIGHT and responsible for overall smooth operations and intelligence behind the decision making. iLIGHT Server can be installed on premises on a blade server in a data centre or on a highspeed Server Machine operating on Windows or Linux Platform. Note that iLIGHT Server can be installed via an Installer on latest Windows or Linux Operated Server. iLIGHT Server Application Software constitutes of following Software Modules.

$>$ iLIGHT Controller : It is the main controller controlling the operation of iLIGHT Server and keeps all the modules of iLIGHT interconnected and informed. Based on the information that comes from various modules iLIGHT Controller taken the appropriate decisions maintains the database, communicates the decisions to various LNUs, keeps track of warnings, alerts, and notifications. It is also responsible to grant communication access to various iLIGHT users and users who connected via web and or mobile app.

> GUI : Graphical User Interface is a local user interface provided for iLIGHT Server users. Users can directly login to iLIGHT Server through valid credentials and can access various information of iLIGHT Server dedicated for iLIGHT Users such as administrative users and Power Users. No Guest User account can be possible with iLIGHT due to sensitive information security and relevant control restrictions. GUI facilitates following

$>$ User dashboard to iLIGHT Admin and Power Users 
$>$ Presents Health and Monitoring Information of iLIGHT

$>$ Data of selected LNUs

$>$ Configuration Settings of iLIGHT

$>$ Information related to various connected users over Web and Mobile App

$>$ Help Information related to iLIGHT Controls and settings

$>$ Data Encryption Module: Encryption module is responsible for decryption of incoming communication packets and decrypting of outgoing communication packets from iLIGHT Controller. The encryption module by default uses SHA-256 algorithm for encrypting the communication messages which needs to be transmitted from iLIGHT Server to LNU and also the data which needs to be stored in iLIGHT Database. iLIGHT Controller provides user configurable settings to encrypt the messages also using MD5 or SHA0/SHA1/SHA-224 algorithms.

$>$ Video and Image Processing Engine: This module is responsible for processing incoming selected video and image footage from various LNUs, process this data, filter the useful contents, reject the unwanted data, time stamped the data along with location coordinates and store the relevant data in iLIGHT Database for offline analysis at a later stage. This module also detects the emergency situations if any based on the analysis of video and image footage and instructs the Alarms module to generate the emergency alarms or provides recommendations to change illumination settings of various LNUs based on the real geographic situations and surrounding environmental conditions.

$>$ Data Analytics Module: Data Analytics module runs various analytics algorithm on the received data from various LNUs, and Video and images data. Data analytics module carries out following selected data analytics algorithms.

$>$ Data filtering

$>$ Data Processing

$>$ Min/Max/Average Data value calculations

$>$ Identification of various Data Trends

$>$ Identifying abnormal Data

$>$ Calculations of Data Trends

$>$ Calculations of Seasonal data variations and correlation

$>$ Data correlation among various data parameters

Based on various data analysis algorithms, Data Analytics Module serves the following purposes

$>$ Predictive Data Analysis

$>$ Diagnostics Data Analysis

$>$ Energy Savings Calculations

$>$ Power Cost Calculations

$>$ Peak Power Predictions

$>$ Failure Analysis

$>$ LED Lights switching analysis

$>$ Seasonal Illumination Analysis

$>$ Generates relevant Alarms based on situational data analysis

$>$ Alarms and Notifications Handler: This module as name suggests serve the purpose of generating of various Alarms, Warnings and Notifications. The generated Alarms/Warnings/Notifications are then sent to GUI for display purpose and or to respective LNUs for necessary actions based on the decisions from iLIGHT Controller. This module also generates various Events related data and logs it for offline analysis and audit purpose.

$>$ Communication Handler: This module is responsible for handling all the internal and external communication from iLIGHT Server. The Module communicates with Internet Gateway to send and receive the communication packets from and to iLIGHT Server. This module communicates with all the modules of iLIGHT Server and prepares various communication packets based on iLIGHT Controller instructions and transmits it to Internet Gateway as per requirements. It also receives the incoming communication packets, processes it, and sends the process data to various modules of iLIGHT Server as per requirements. It also handles the errors if any during the communication. 


\section{3 iLIGHT Client}

These are iLIGHT registered users, known as clients, who can connect to iLIGHT Server over HTTP/HTTPS connection from their system web browser over the internet. The iLIGHT Clients will be various stakeholders from Smart City who wants to avail iLIGHT provided information for various purposes. Following stakeholders can be iLIGHT Clients.

$>$ iLIGHT Admin and Power Users

$>$ Street Light Maintenance Staff

$>$ Smart City Admin Staff

$>$ Smart City Lighting Services Staff

$>$ Traffic Controllers

$>$ Smart City Environment Management Staff

iLIGHT Client webpages are served by iLIGHT Server and facilitate the following based on privilege information accessible to various users according to their granted rights

$>$ User Dashboard

$>$ Alarms and Notifications

$>$ Emergency Alerts

$>$ Data Logs and Graphs, Events Logs

$>$ Maintenance Schedule for various Street Lights/ Geography Lights

$>$ Various Data Analytics Reports and Information with respect to Power Savings, Peak Power Requirements, Power needed, Total Power consumed for a stated period, Power Cost,

$>$ Real Time Video Footage and images of particular Lamppost and or Street Lights

$>$ Various Configuration Settings

\section{4 iLIGHT Mobile App}

iLIGHT Mobile App facilitates the iLIGHT Clients to connect to the iLIGHT Server through Mobile. iLIGHT Mobile App is a lightweight GUI provided for iLIGHT Clients over Mobile. iLIGHT Mobile App supports both Android and IOS mobile operating system. iLIGHT Mobile App supports all the user interfaces and functionalities available for iLIGHT clients over the Web. The only difference is in look and feel of GUI which is basically made compatible for Mobile Users.

\subsection{Internet Infrastructure}

This infrastructure is responsible for providing wired or wireless connectivity to an Internet and various subsystems of iLIGHT Solution. The infrastructure contains Gigabit Speed fast Internet connection, various routers, Internet Gateway, Wireless Routers, free wireless hotspots etc. This infrastructure need not be separately arranged, instead it is an existing infrastructure of Smart City which can be effectively used to interconnect iLIGHT Solution components to internet. Since iLIGHT Solution basically works on backbone of highspeed Internet connection, Internet Infrastructure is one of the most important part of iLIGHT Solution.

\section{ILIGHT - ADVANTAGES AND LIMITATIONS :}

iLIGHT Solution is proposed after study and analysis of existing Smart Lighting Systems used in Smart Cities and based on feedback and suggestions from various stakeholders from Smart Cities, researchers, and Industry SMEs. While proposing the iLIGHT Solution, due care is taken to understand some of the key limitations in existing Smart Lighting System and how we can remove such deficiencies. iLIGHT Solution framework is subsequently discussed with some stakeholders including industry SMEs and Smart City Engineers and evaluated at laboratory level using limited scale simulated data under various test scenarios by certain research scholars and with this limited evaluation the results obtained were encouraging. Based on the results obtain it is confirmed that some of the key advantages of proposed iLIGHT Solution are as follows.

- The entire system is modular and hence it is easy to assemble and deploy

- The proposed system is based on Commercial Off the Shelf (COTS) industrial grade components and hence will be comparatively economical

- Troubleshooting and maintenance of the system is easy since the system is equipped with predictive diagnostics and maintenance alarms as per requirement and hence due maintenance can be taken care of well on time. 
- The user dashboard for various stakeholders is available over the Web as well as through Mobile App, making user interface very easy and operatable through remote

- Can be easily integrated with existing IT Infrastructure and Services of Smart City

- Highly encrypted message communication, makes the system robust and safe from any vulnerability and cyber attacks

- Storage and retrieval of key parameters data can be used for offline analysis and audit purpose, makes the operation of the system very transparent

- Various illumination settings, light dimming features and automated switching of LED Lights as per seasonal variations, movement detections and environmental conditions makes it very efficient and allows maximum power savings.

- Remote operation and management make it safe to operate

- Support to get power from renewable energy sources makes it power requirement very economical and operating cost lower.

Though proposed iLIGHT Solution offers safe, reliable, and economical operating Smart Lighting System, based on the analysis of results obtained from iLIGHT Solution evaluation, it is found that iLIGHT Solution may have certain limitations depending on its configuration settings. Such limitations can be as follows

- If iLIGHT is configured to use SHA-256 encryption algorithm for all the message communications including Video and Image data then it can require huge storage database and also frequent backup of data, besides its operation speed may lag in certain cases where large size communication packets can take more time for message decryption and may require very high bandwidth of Internet.

- The operation of iLIGHT is heavily dependent on reliable Internet connection, if the Internet connection is not stable and or not offering the right speed and bandwidth, then it will directly impact the performance of iLIGHT

- If iLIGHT Server is installed on on-premises high speed server instead of on the cloud, then initial investment cost of such system can be higher

\section{RESULTS AND DISCUSSION :}

The limited evaluation of iLIGHT Solution Framework through stakeholder discussion and simulated model testing at laboratory level using simulated data, provided very encouraging results. The results obtained are tested at multiple levels and multiple iterations and the summary of results is as per the following table.

Table 3: Broad Evaluation Results for ILIGHT Solution

\begin{tabular}{|c|c|c|c|}
\hline $\begin{array}{l}\text { Sr. } \\
\text { No. }\end{array}$ & Parameter & Result/Outcome & Remarks \\
\hline 1. & Cost & Comparatively Lower & Due to use of Off the Shelf Components \\
\hline 2. & $\begin{array}{l}\text { Development } \\
\text { and Maintenance }\end{array}$ & $\begin{array}{l}\text { Less time consuming } \\
\text { and easy }\end{array}$ & $\begin{array}{l}\text { Due to Modular Architecture, assembly and } \\
\text { maintenance is easy. Due to predictive } \\
\text { failure analysis, it is possible to detect } \\
\text { possibility of failure well ahead of time, } \\
\text { also due to scheduled maintenance alerts, } \\
\text { maintenance is easy }\end{array}$ \\
\hline 3. & Oper & Faster and Economical & $\begin{array}{l}\text { Due to use of High-Speed Ethernet } \\
\text { communication. Also, the existing IT } \\
\text { Infrastructure can be used for installation } \\
\text { and deployment }\end{array}$ \\
\hline 4. & Coml & Less Complex & Due to Modular Architecture \\
\hline 5. & User Experience & Much Better & $\begin{array}{l}\text { Due to configurable User Dashboard for the } \\
\text { Clients connecting over the web as well as } \\
\text { through mobile app }\end{array}$ \\
\hline 6. & Energy Savings & $\begin{array}{l}\sim 30 \text { to } 40 \% \text { more } \\
\text { compare to existing } \\
\text { systems }\end{array}$ & $\begin{array}{l}\text { Due to capability of effective illumination } \\
\text { control based on seasonal variations, } \\
\text { environmental conditions, dimming control }\end{array}$ \\
\hline
\end{tabular}




\begin{tabular}{|c|c|c|c|}
\hline & & & $\begin{array}{l}\text { and switching based on analysis of real } \\
\text { video/image footages }\end{array}$ \\
\hline 7. & $\begin{array}{l}\text { Application } \\
\text { Scope }\end{array}$ & Wider & $\begin{array}{l}\text { Can be successfully used for Street } \\
\text { Lighting, Playgrounds Lightings, Garden } \\
\text { and Lawn Lightings, for Smart Building } \\
\text { Lightings, Offices and Home Lighting and } \\
\text { all commercial areas including markets and } \\
\text { highway lighting }\end{array}$ \\
\hline 8. & Feature Support & Widest & 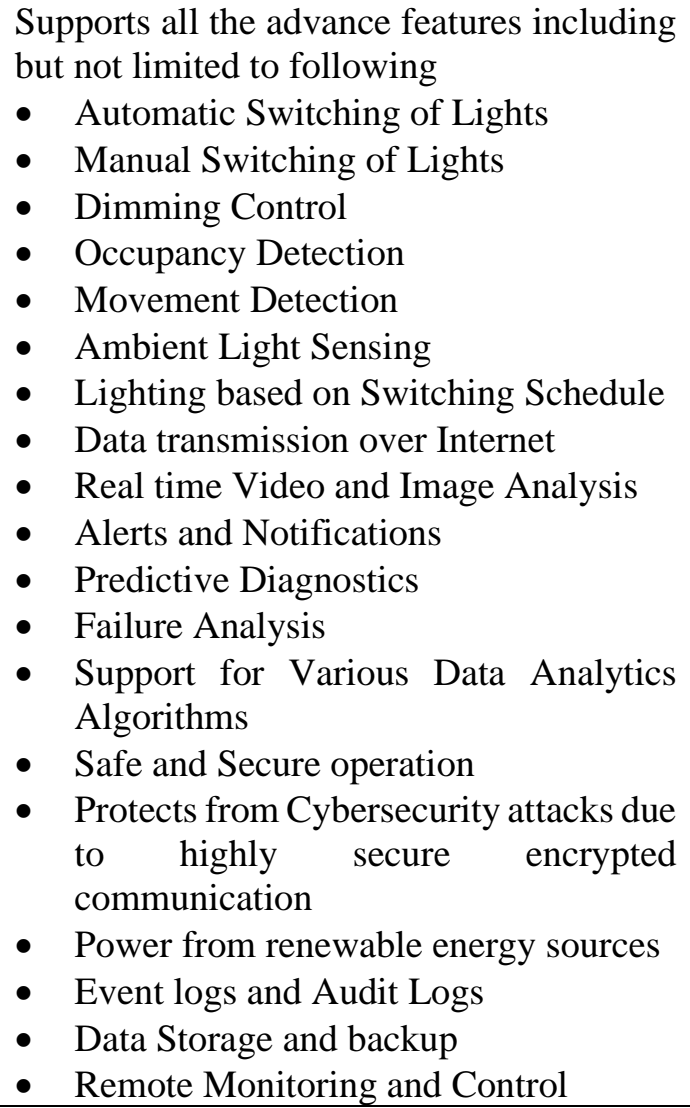 \\
\hline
\end{tabular}

In summary, iLIGHT Solution makes use of the modern infrastructure of Smart Cities, the widest range of Sensors and high-speed Server and Application Software at the Node level and at Central Server level and with the help of efficient data analytics algorithms, can offer the widest range of features and functionalities which goes well beyond automatic switching of LED Lights to failure analysis, predictive diagnostics to efficient power savings.

\section{CONCLUSION :}

Smart Lighting is an essential service of Smart Cities. It plays a crucial role in supplying lighting as per the requirement of Smart City stakeholders. A smart Lighting System is an advanced Lighting System used by Smart Cities for efficient lighting supply at a reasonable cost. It also saves power without making any compromise on lighting quality. Smart Lighting not only provides lighting but also serves the purpose of connecting various devices over the internet and data transmission across connected devices. While selecting and deploying Smart Lighting System across Smart Cities, the operating and maintenance cost also needs to be considered since Smart Lighting System is a complex system and may not be easy to operate and maintain. The proposed "iLIGHT" Smart Lighting Solution framework is designed keeping in mind, not only the parameters such as Lighting efficiency, energy-saving, power cost, etc. but also the requirements such as less costly, easy to deploy, operate, and maintain.

\section{FUTURE WORK :}

This paper has discussed the conceptual framework for Smart Lighting Solution "iLIGHT" for Smart Cities, its typical architecture, features, key components, advantages, and the limitations. The conceptual framework is proposed with an intention to serve the ready reference and design information to assist the stakeholders of Smart City to design and or choose the right lighting solution for the Smart 
Cities. Since the proposed "iLIGHT" solution is still available on paper, in the future, I am planning to undertake its implementation to understand and evaluate its features, benefits, and operation deficiencies if any, in more details. A separate research paper will be prepared on this to share the "iLIGHT" performance data, its evaluation results and overall feedback from various stakeholders after using the implemented system.

\section{REFERENCES :}

[1] Gade, Dipak S. (2019). Introduction to Smart Cities and Selected Literature Review. International Journal of Advance and Innovative Research, 6(2), 7-15.

[2] CBS Chicago. The city is saving $\$ 10$ million in electricity costs every year with LED streetlights. Retrieved from https://chicago.cbslocal.com/2020/02/17/city-says-its-saving-10-million-a-yearwith-new-led-streetlights-but-structural-integrity-of-poles-is-not-being-checked/ on September 01,2021

[3] Gade, Dipak S. (2019). Technology Trends and Digital Solutions for Smart Cities Development. International Journal of Advance and Innovative Research, 6(1), 29-37.

[4] M. H. Toufiq Imam, Sina Afshari and Sandipan Mishra, (2018). Smart Lighting control system. Intelligent building control system (221-251), Springer International Publishing.

[5] Amit Kumar Sikder, Abbas Acar, et al. (2018). IoT-enabled Smart Lighting Systems for Smart Cities. IEEE 8th Annual Computing and Communication Workshop and Conference (CCWC), 639645. doi: 10.1109/CCWC.2018.8301744.

[6] Giuseppe Cacciatore, Claudio Fiandrino, Dzmitry Kliazovich, Fabrizio Granelli, Pascal Bouvry, (2017). Cost Analysis of Smart Lighting Solutions for Smart Cities. IEEE ICC Green Communications Systems and Networks, 1-6.

[7] Marc Füchtenhans, Eric H. Grosse \& Christoph H. Glock, (2021). Smart lighting systems: stateof-the-art and potential applications in warehouse order picking. International Journal of Production Research, vol. 59(12). 3817-3839.

[8] Thomas van de Werff, Harm van Essen, Berry Eggen, (2017). The impact of the internet of lighting on the office lighting value network. Journal of Industrial Information Integration. 11(1), 29-40.

[9] Baoshi Sun, Qiaoli Zhang and Shi Cao, (2020). Development and Implementation of a SelfOptimizable Smart Lighting System Based on Learning Context in Classroom. International Journal of Environmental Research and Public Health, 17(1), 1-26.

[10] Yusi Cheng, Chen Fang, Jingfeng Yuan, and Lei Zhu, (2020). Design and Application of a Smart Lighting System Based on Distributed Wireless Sensor Networks. MDPI Applied Sciences, 10(1), $1-21$.

[11] Tran Anh Khoa, Le Mai Bao Nhu, et al. (2020). Designing Efficient Smart Home Management with IoT Smart Lighting: A Case Study. Hindawi Wireless Communications and Mobile Computing, 2020(1), 1-18.

[12] Eisley Dizon and Bernardi Pranggono, (2021). Smart streetlights in Smart City: a case study of Sheffield. Journal of Ambient Intelligence and Humanized Computing, 1-26. DOI: 10.1007\%2Fs12652-021-02970-y.

[13] Naser Hossein Motlagh, Mahsa Mohammadrezaei and Julian Huntand Behnam Zakeri, (2020). Internet of Things (IoT) and the Energy Sector. MDPI Energies, 13(1), 1-27.

[14] Sarah Faltaous,Salma Eljaki, Stefan Schneegass, (2019). User Preferences of Voice Controlled Smart Light Systems. Association for Computing Machinery, 1-6.

[15] Gade, Dipak S. (2021). Disruptive Technologies for Efficient and Sustainable Smart Cities. International Journal of Management, Technology, and Social Sciences (IJMTS), 6(2), 48-63.

[16] Leonardo Guevara and Fernando Auat Cheein, (2020). The Role of 5G Technologies: Challenges in Smart Cities and Intelligent Transportation Systems. MDPI Journal, Sustainability, 12(6469), $1-15$. 
[17] Enrico Buzzoni, Fabio Forlani, et al. (2019). The Advent of the Internet of Things in Airfield Lightning Systems: Paving the Way from a Legacy Environment to an Open World. MDPI Sensors, 19, 1-13.

[18] Gade, Dipak S., \& Aithal, P. S., (2021). Smart Cities Development During and Post COVID-19 Pandemic - A Predictive Analysis. International Journal of Management, Technology, and Social Sciences (IJMTS), 6(1), 189-202.

[19] Firdaus, R. and Mulyana, E. (2017). Smart Building Lighting System. IOP Conf. Series: Materials Science and Engineering, 384, 1-8.

[20] Naser Hossein Motlagh, Mahsa Mohammadrezaei, Julian Huntand and Behnam Zakeri, (2020). Internet of Things (IoT) and the Energy Sector. MDPI Energies, 13(494), 1-27.

[21] Gade, Dipak S. \& Aithal, P. S. (2020). Blockchain Technology: A Driving Force in Smart Cities Development. International Journal of Applied Engineering and Management Letters (IJAEML), 4(2), 238-252.

[22] Paul Golata, Connected Intelligent LED Lighting Networks: Intelligent Lighting Integrated Within Smart Buildings. Retrieved from https://www.mouser.in/applications/connected-intelligent-ledlighting-networks/ on September 01, 2021.

[23] On The Value of Street Lamp System in Smart City. Retrieved from https://www.zgsmlightings.com/news/on-the-value-of-street-lamp-system-in-smart-ci14180805.html on September 01, 2021.

[24] Gianfranco Gagliardi, Marco Lupia, et al. (2020). Advanced Adaptive Street Lighting Systems for Smart Cities. Smart Cities, 3(4), 1495-1512. 